\section{Upper GI Obstruction Presenting as Failure to Thrive: A Case of Achalasia}

\section{Sir,}

Achalasia is characterised by impaired esophageal motility and inability of the lower esophageal sphincter (LES) to properly relax in response to swallowing. ${ }^{1}$ It is rare in children and even more infrequent in children $<5$ years of age. The incidence in childhood is $0.11 / 100,000$ children annually. It is frequently misdiagnosed as gastroesophageal reflux disease (GERD); and prior to its diagnosis, up to $50 \%$ of children are treated with antacids or prokinetics. ${ }^{2}$

A 3-year male child presented with chief complains of fever, productive cough, and vomiting since 10 days. Vomiting was non-projectile, and occurred after feeds. The child had been previously admitted with same complaints. Once, it was diagnosed as failure-to-thrive secondary to GERD; and second time as protein caloric malnutrition (PCM) and persistent vomiting. He had received multiple doses of antacids, antiemetics and multivitamins, but to no avail. Patient was born as a full-term baby with delayed cry. Vaccination was up to date. There was gross motor delay. Neck holding was achieved at six months and sitting at two years; and till now, he was standing with support but walking had not been achieved. He had three other normal siblings. Family history was not significant. Baby was mother-fed for a year and then started on formula milk. Weaning was delayed up to a year and was abrupt and improper as patient had difficulty swallowing. By the time patient reached us, he weighed $6 \mathrm{kgs}$ at three years (below $3^{\text {rd }}$ centile).

Serum sodium was $126 \mathrm{mmol} / \mathrm{l}$ (normal=135-150 mmol/l), potassium, $2.1 \mathrm{mmol} / \mathrm{l}$ (normal=3.5-5.1 mmol/l), chloride, 94 $\mathrm{mmol} / \mathrm{l}$ (normal= 96-106mmol $/ \mathrm{l}$ ), calcium, $8.8 \mathrm{mmol} / \mathrm{L}$, and serum creatinine was $22 \mu \mathrm{mol}$. Workup for celiac disease was normal. Arterial blood gases (ABGs) showed a pH of 7.5 (normal= 7.35-7.45). Complete blood count (CBC) was normal with all cell lines in the normal range. Serum bicarbonate was 23 $\mathrm{mmol} / \mathrm{l}$ (normal=21-28 mmol/l) and $\mathrm{pCO}_{2}$ was $25 \mathrm{~mm}$. Repeat electrolytes and ABGs showed sodium to be $128 \mathrm{mmol} / \mathrm{l}$, potassium, $2.7 \mathrm{mmol} / \mathrm{l}$, chloride, $100 \mathrm{mmol} / \mathrm{l}$, bicarbonate, 30.8 $\mathrm{mmol} / \mathrm{l}$ and $\mathrm{pH}, 7.6$. Patient had films, and report of barium meal advised by a general pediatrician (Figure 1), which showed no abnormalities. As symptoms and signs had worsened over time, so barium swallow was done with thick and thin barium solution. Barium outlined the pharynx, hypopharynx, esophagus and the gastroesophageal junction. Thoracic part of the esophagus was moderately to massively dilated. Smooth narrowing was noted at the lower end of the esophagus secondary to stricture formation or a possible atretic segment. Patient was referred to pediatric surgeon (Figure 2).

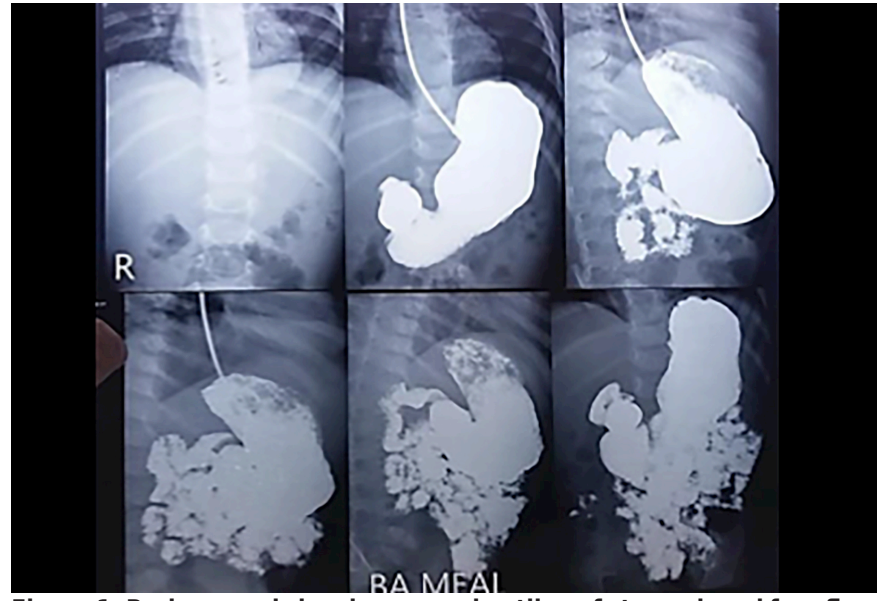

Figure 1: Barium meal showing normal outline of stomach and free flow intoduodenum.

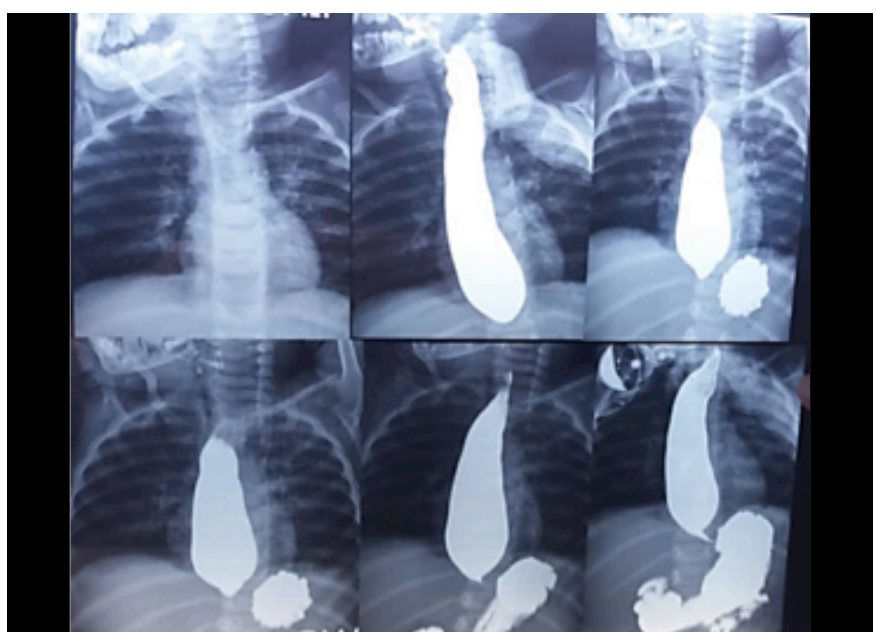

Figure 2: Barium swallow showing gross dilation of esophagus and classic rat-tail sign of achalasia. There is delay in passage of contrast to stomach.

Some common causes of recurrent vomiting in children are acute viral gastroenteritis, GERD, pyloric stenosis, intestinal obstruction (e.g., meconium ileus, malrotation with volvulus, congenital bands, intestinal atresia or stenosis), and intussusception. Less common causes include sepsis and food intolerance. Metabolic disorders (e.g., urea cycle disorders, organic acidemias) are uncommon but can manifest with vomiting. ${ }^{3}$

Achalasia results from inadequate LES relaxation in the absence of distal mechanical obstruction. ${ }^{4}$ It may be a result of absent or impaired inhibitory innervations in myentric plexus or it may be secondary to GERD, Chagas disease, or viral infection. ${ }^{2,3}$ Achalasia has been associated with various syndromes like achalasia, alacrima, and ACTH insensitivity (AAA) syndrome, progressive cerebellar ataxia, parkinsonism, familial glucocorticoid deficiency, mental retardation, and Down syndrome. Commonest symptoms include dysphagia, vomiting or regurgitation, retrosternal pain, failure-to-thrive and respiratory symptoms due to chronic aspiration. It is often confused with and treated wrongly as GERD, because the common trait of achalasia is the emesis of uncurdled milk in breastfeeders, which issimilarto GERD. ${ }^{1-3}$ Achalasiacan be diag- 
nosed with barium swallow and esophageal manometric studies. A barium study reveals a dilated esophagus with a bird's beak (rat-tail) appearance at the distal end. Moreover, manometric esophageal evaluation exhibits elevated resting LES pressure, absent or low-amplitude peristalsis, or non-relaxing LES. In addition, upper endoscopy and biopsy of the distal end of esophagus is also performed to rule out secondary causes, e.g. malignancy. ${ }^{4}$ Endoscopy often demonstrates a tight LES, retained saliva and food; however a normal study does not exclude the diagnosis. Treatment modalities include pharmacological and surgical therapies but the prognosis is quite variable in pediatric population. Calcium channel blockers and Botox injections are used in adults frequently; however, doses and frequencies and effects of these drugs in children are not well studied. ${ }^{2}$ Pneumatic dilatations have been used in children and often require multiple dilatation attempts to provide positive results. In children, surgery is the definitive treatment of choice, i.e. Heller's myotomy with or without fundoplication. A new and less invasive therapeutic approach is developed, i.e. POEM (per oral endoscopic myotomy), which has been used in both adults and children and exhibits equally effective results; however, its use is still limited in pediatric achalasia. ${ }^{4,5}$ Owing to its presentation and the fact that it can lead to failure-to-thrive, the disease should be considered an important, though a rare differential diagnosis for persistent vomiting in children.

\section{CONFLICT OF INTEREST:}

The authors declared no conflict of interest.

\section{AUTHORS' CONTRIBUTION:}

SG: Conceptualisation, patient diagnosis,write-up, literature search, and proofreading.
AU: Patient diagnosis, workup, and literature search. SFMS: Patient diagnosis, workup, and proofreading.

\section{REFERENCES}

1. Franklin AL, Petrosyan M, Kane TD. Childhood achalasia: A comprehensive review of disease, diagnosis and therapeutic management. World J Gastrointest Endosc 2014; 6(4):105-11. doi:10.4253/wjge.v6.i4.105.

2. Reynolds JC, Parkman HP. Achalasia. Gastroenterol Clin North Am 1989; 18:223-55.

3. Mullen N. Pediatric health .Vomiting in the pediatric age group. Disclosures. Pediatr Health 2009; 3(5):479-503.

4. Krill J, Naik R, Vaezi M. Clinical management of achalasia: current state of the art. Clin Exp Gastroenterol 2016; 9:71-82. doi.org/10.2147/CEG.S84019.

5. Lee CW, Kays DW, Chen MK, Islam S. Outcomes of treatment of childhood achalasia. J Pediatr Surg 2010; 45:1173-7. doi: 10.1016/j.jpedsurg.2010.02.086.

Saima Gillani, Aziz Ullah and Syed Faiz Muhammad Shah

Department of Pediatrics, Ayub Medical College, Abbottabad, Pakistan

Correspondence to: Dr. Saima Gillani, Department of Pediatrics, Ayub Medical College, Abbottabad, Pakistan E-mail: drsaimagillani@gmail.com

Received: July 24, 2020; Revised: September 05, 2020; Accepted: November 07, 2020

DOI: https://doi.org/10.29271/jcpsp.2021.07.881 